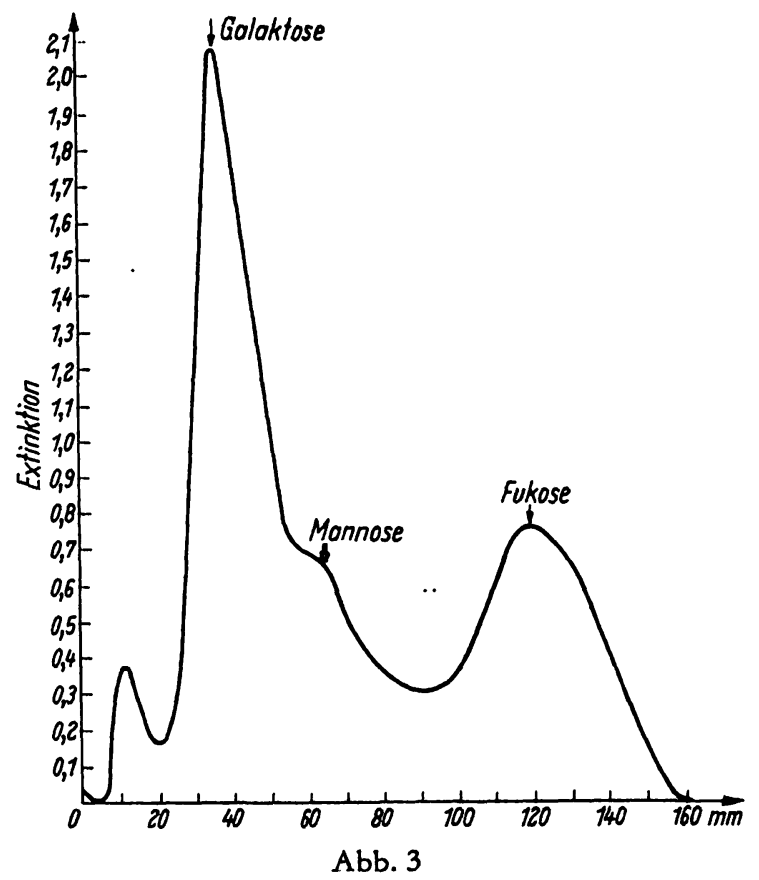

Chromatogramm bei atrophischer Gastritis

Hydrolyse und Chromatographie s. Legende Abb. 1. - Anilinphthalat-Reagenz; Auswertung im Spektralphotometer

Glukuronsäure, die sehr hitzeempfindlich ist, im menschlichen Magensaft von der Methode der Hydrolyse abhängig. Der Nachweis von Glukuronsäure gelingt, wenn man die chromatographisch als Glukuronsäure erkannte Bande mit Wasser eluiert und das Eluat nach Tolnens mit Naphthoresorcin und rauchender Salzsäure versetzt.

Quantitative Aussagen sind bei papierchromatographischen Analysen problematisch, da die verschiedenen Zuckerkomponenten sich nicht quantitativ anfärben. Unsere Ergebnisse bei einer Untersuchung von normaler Magenschleimhaut und Gastritis zeigen für den rel. $\%$-Gehalt der Aminozucker nach der $\chi^{2}$-Methode eine Tendenz, die nahezu die 5\%-Wahrscheinlichkeitsgrenze erreicht. Danach muß im Sekret bei normaler Magenschleimhaut ein niedrigerer Aminozuckergehalt erwartet werden, was sich auch mit den Befunden von Grass $(10,11)$ deckt. Einen erhöhten Aminozuckergehalt wird man bei einer chronischen Entzündung und vor allem bei der atrophischen Gastritis erwarten können. Eine verbesserte Methodik der Magensaftanalysen ist notwendig, um zu endgültigen Aussagen zu gelangen. Hinsichtlich der Aminozucker ist zu beachten, daß ein Teil der Hexosamine an den Ionenaustauscher quantitativ adsorbiert wird. Inwieweit Aminozucker sich durch Zugabe von Säure zum Magensafthydrolysat vom Ionenaustauscher eluieren lassen, um zu einem vollständig quantitativen Nachweis zu kommen, ist noch nicht restlos geklärt.

\title{
Literatur
}

1. Preisser, F., G. Berg und N. Henning, Zschr. Gastroenterol. 1, 155 (1963). - Berg, G. und F. Preisser, Gastroenterologia, Basel, Suppl. zu 97, 238 (1962). - 3. Berg, G., N. Henning, K. Heinkel und W. Lentzen, Klin. Wschr. 38, 282 (1960). 4. Clotren, R. und A. Clotren, Hochspannungselektrophorese, Georg Thieme Verlag, Stuttgart (1962). - 5. HAIs, J. M. und K. Macek, Handbuch der Papierchromatographie, Bd. I, Gustav Fischer Verlag, Jena (1958). - 6. CRAMER, F., Papierchromato- graphie, Verlag Chemie GmbH, Weinheim/Bergstr. (1962). 7. Deutsch, E., H. J. Christian und N. A. Peloquin, Amer. J. Gastroenterologie 41, 467 (1964). - 8. Schrager, J., Nature (London) 198, 899 (1963). - 9. Schrager, J., Nature (London) 201, 702 (1964). - 10. Glass, G. B. J., M. Rich und L. Stephanson, Gastroenterology, Baltimore 34, 598 (1958). - Glass, G. B. J., L. Stephanson-Liounis, M. Rich und S. E. Mitschell, Proc. Wld. Congr. Gastroenterol. 1958 994, Baltimore.

Privatdozent Dr. med. G. Berg

Med. Univ.-Klinik, 852 Erlangen, Krankenhausstr. 12

\section{Die Fraktionierung von Pleurapunktat-Proteinen im Zusammenhang mit der Bestimmung von Entzündungsaktivitäten}

\author{
Von J. WAGNER und B. SCHMIDT
}

Aus der Medizinischen Klinik der Karl-Marx-Universität Leipzig (Direktor: Prof. Dr. R. Emmrich)

Herrn Prof. Dr. Dr. h. c. Dr. h. c. Dr. h. c. Dr. h. c. K. Mothes zum 65. Geburtstag

(Eingegangen am 23. August 1965)

\begin{abstract}
Die Pleurapunktat-Proteine wurden mit verschiedenen „Sephadex“-Typen fraktioniert, wobei mit Sephadex A-25 6 verschiedene Fraktionen erhalten wurden. Die mit dem Trypanblau-Test durchgeführten Bestimmungen der Entzündungsaktivität waren in den Fraktionen positiv, die neben Albumin $\alpha_{1^{-}}$und $\alpha_{2}$-Globuline enthielten. Diese Ergebnisse konnten durch eine fraktionierte Fällung mit Ammoniumsulfat bestätigt werden.

The proteins from pleural puncture were fractionated with various types of Sephadex; six fractions were obtained with Sephadex A-25. Tests for inflammatory activity with trypan blue were positive for those fractions which ran close to albumin, $\alpha_{1}$-and $\alpha_{2}$-globulins. These results were confirmed by fractionation with ammonium sulphate.
\end{abstract}

Die Exsudation bei der akuten Entzündung wird nach LEwIs (1) hauptsächlich durch Freisetzen der in den Mastzellen befindlichen stark vasoaktiven Stoffe hervorgerufen. Relativ geringe mechanische, thermische, elek- trische und aktinische Reize führen zu einer sofortigen Entgranulierung dieser Zellen mit Abgabe ihrer Inhaltsstoffe. Neben dem Histamin und dem Heparin spielen seit einigen Jahren kreislaufwirksame Polypep- 
tide, die sogenannten Plasmakinine, eine wesentliche Rolle. Es ist nicht ausgeschlossen, daß es neben den bekannten Kallidin und Bradykinin noch weitere Kinine gibt, und es ist auch denkbar, daß die Menkinschen Stoffe (Leukotaxin, Exsudin, Nekrosin usw.) (2) Plasmakinine darstellen (3).

$\mathrm{Da}$ diese „Entzündungsstoffe“ keine einheitlichen Substanzen darstellen und teilweise chemisch nicht näher untersucht sind (2), erschien es angebracht, die Pleurapunktat-Proteine mit Hilfe moderner analytischer Methoden aufzutrennen.

Die Chromatographie an quervernetzten Dextrangelen (Sephadex, AB Pharmacia, Uppsala/Schweden) (4) hat sich auf Grund ihres Trennprinzips nach der Molekülgröße und zusätzlich durch den Einbau von Ionengruppen für die schonendste Trennung von Proteingemischen bewährt (5). Diese Methode bot sich vor allem auch deswegen an, weil die Elution mit Kochsalzkonzentrationen vorgenommen werden kann, die um den physiologischen Wert liegen. Die Bestimmung der Entzündungsaktivität der einzelnen Fraktionen konnte deshalb ohne Schwierigkeiten am Kaninchen durchgeführt werden.

\section{Methodik}

Zur Untersuchung gelangten in sämtlichen Fällen Pletıraexsudate verschiedener Pathogenese. Der Proteingehalt der Punktate betrug 2,8 bis 5,1g\%.

\section{Ammoniumsulfat-Fraktionierung}

Eine grobe Fraktionierung der Pleurapunktat-Proteine wurde durch Fällung mit Ammoniumsulfatlösung unterschiedlicher Konzentration erreicht (6). Dazu wurden in $5 \mathrm{~m} l$ eines Punktates durch tropfenweise Zugabe der fünffachen Menge einer 34-proz., 40-proz., 50-proz. und 60-proz. Ammoniumsulfatlösung die Eiweiße ausgefällt. Nach einer Stunde wurden die entsprechenden Niederschläge abzentrifugiert und in $5 \mathrm{~m} l$ Kochsalzlösung gelöst. Nach Dialyse gegen physiologische Kochsalzlösung wurden die einzelnen Fraktionen in vivo ausgetestet.

\section{Chromatographie an Dextrangelen}

Für die Trennung der Pleurapunktat-Proteine wurde Sephadex G-25, G-50, G-75, G-100, G-200 und Sephadex A-25 und A-50 verwendet. Die G-Typen wurden in physiologischer Kochsalzlösung gequollen und durch mehrmaliges Waschen der sehr feinkörnige Anteil durch Dekantieren entfernt.

Die A-Typen wurden zuerst 24 Stunden mit Aqua dest. gequollen. Danach wurden kleinere Gelpartikel durch wiederholtes Dekantieren abgetrennt und das gequollene Gel mit $0,5 n-\mathrm{HCl}$ behandelt. Nach öfterem Waschen mit Aqua dest. erfolgte die Behandlung des Austauschers mit $0,5 n-\mathrm{NaOH}$. Zur Entfernung der überśchüssigen Ionen wurde nochmals gründlich mit Aqua dest. gewaschen und danach das Gel mit 0,035 $\mathrm{m} \mathrm{NaCl}$-Lösung oder einem entsprechenden Puffer äquilibriert. Mit den so präparierten Gel-Suspensionen wurden die Chromatographie-Säulen gefüllt. Durch Heben und Senken des Ge- fäßes mit der Elutionslösung konnte der gewünschte hydrostatische Druck eingestellt werden, so daß die Durchflußgeschwindigkeiten bei den G-Typen $60 \mathrm{~m} / \mathrm{Std}$. und bei den A-Typen $40 \mathrm{~m} / / \mathrm{Std}$. betrugen. Das Eluat wurde in Fraktionen zu $5 \mathrm{~m} l$ aufgefangen. Sämtliche Proteintrennungen wurden bei einer Temperatur von $10^{\circ} \mathrm{C}$ durchgeführt. Die Messung der Lichtabsorption erfolgte bei $280 \mathrm{~m} \mu$.

\section{Papierelektrophorese}

Die dem Kurvenbild der Lichtabsorption entsprechenden Proteinfraktionen der Gel-Filtration und auch der Ammoniumsulfatfällungen wurden nach $L$ yophilisierung papierelektrophoretisch . untersucht. Die Trennung geschah auf FN4-Papier der Firma Spezialpapierfabrik, Niederschlag/Erzgeb. Als Puffer diente Veronal-NatriumPuffer $\mathrm{pH}=8,6$. Die Laufzeit betrug 16 Stunden bei einer Spannung von 4 Volt $/ \mathrm{cm}$. Das Anfärben der Eiweißfraktionen geschah nach Grassmani und HaNNig (7) mit Amidoschwarz $10 \mathrm{~B}$.

\section{Trypanblau-Test}

Zur Bestimmung der Entzündungsaktivität wurde der sogenannte Trypanblau-Test $(8,9)$ am Kaninchen ausgeführt. Dazu wurden die Bauchhaare von weißen Kaninchen unter Vermeidung von Hautläsionen geschoren und mit einem Enthaarungspulver gänzlich von der Haut entfernt. Danach wurden etwa $0,2 \mathrm{ml}$ einer Proteinfraktion intrakutan injiziert. Nach intravenöser Injektion einer 1-proz. physiologischen Lösung Trypanblau konnten in einem Zeitraum von etwa 30 Minuten Hautquaddeln nachgewiesen werden, die zeigen, daß ein permeabilitätssteigernder Faktor wirksam ist.

\section{Ergebnisse und Diskussion}

Zunächst wurde an Sephadex G-25 mit physiologischer Kochsalzlösung als Elutionsmittel eine Abtrennung der niedermolekularen von den hochmolekularen Substanzen durchgeführt, und zwar an zwei Säulen mit unterschiedlichem Gelbettvolumen (Abb. 1). Die kleinere Säule zeigt dabei eine Auftrennung in eine makromolekulare und zwei niedermolekulare Fraktionen. Der Trenneffekt an der größeren Säule war bedeutend schärfer, und die niedermolekularen Substanzen wurden in drei Fraktionen aufgetrennt. Bei den 16 untersuchten Pleuraex-

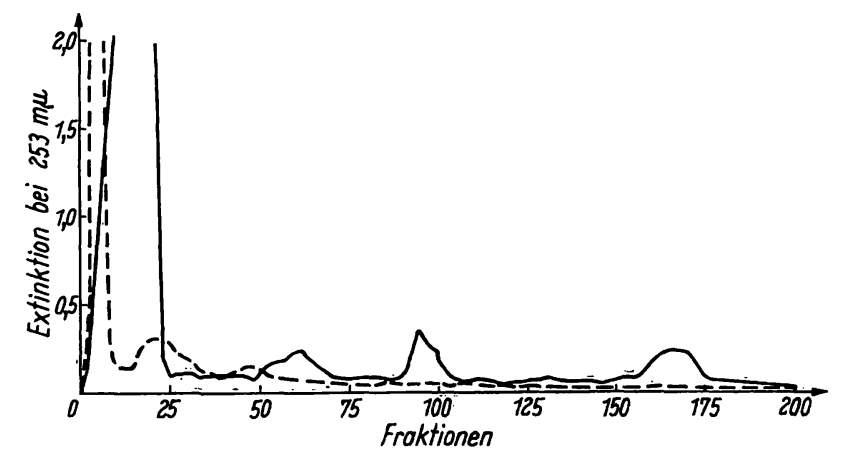

Abb. 1

Trennung von Pleurapunktat-Proteinen an Sephadex G-25.

Säule: $2,2 \times 200 \mathrm{~cm}$; aufgetragene Menge: $25 \mathrm{ml}$ Punktat; - - - Säule: $2,2 \times 40 \mathrm{~cm}$; aufgetragene Menge: $10 \mathrm{ml}$ Punktat; Elutionsmittel: physiol. $\mathrm{NaCl}$-Lösung 
sudaten mit unterschiedlicher Pathogenese war der Trypanblau-Test in der makromolekularen Fraktion stets positiv und in den niedermolekularen Fraktionen stets negativ.

Die Fraktionierung der Pleurapunktat-Proteine an weniger vernetzten Dextrangelen Sephadex G-50, G-75 und G-100 ergab im Hinblick auf die Auftrennung der makromolekularen Anteile keine befriedigenden Ergebnisse. Mit Sephadex G-200 konnte eine Auftrennung der Pleurapunktat-Proteine in 3 Fraktionen erreicht werden. Im folgenden wurde deshalb versucht, eine bessere Trennung an DEAE Sephadex A-25 zu erreichen. Das geschah zuerst mit Hilfe der kontinuierlichen Gradientenelution nach Peterson und Mitarbeitern (10) mit Trispuffer. $\mathrm{Da}$ die erhaltene Auftrennung (Abb. 2) nicht befriedigte, wurde im folgenden mit Hilfe der diskontinuierlichen Gradientenelution mit unterschiedlichen Kochsalzkonzentrationen von $0,035 \mathrm{~m}$ bis $0,38 \mathrm{~m}$ und von $0,1 m$ bis $1,0 m$ eine Auftrennung der Punktat-Proteingemische durchgeführt (Abb. 3). Dabei ist die Elution mit den geringeren Kochsalzkonzentrationen vorzuziehen. Eine Elution mit Hilfe dieser niederen Kochsalzkonzentrationen in Tris- bzw. Phosphatpuffer ergab keine wesentliche Verbesserung des Trenneffektes (Abb. 4). Der Einsatz von Sephadex A-50 führte unter diesen geschilderten Elutionsbedingungen zu keinen besseren Trennungen.

Die erhaltenen Fraktionen (s. Abb. 3) wurden papierelektrophoretisch untersucht. Dabei waren in Fraktion I Albumin, $\alpha_{1^{-}}, \alpha_{2^{-}}, \beta$ - und $\gamma$-Globuline vorhanden. Die Fraktionen II und III enthielten $\gamma$-Globuline; in Fraktion IV und V waren die Albumine, $\alpha_{1}$ - und $\alpha_{2}$-Globuline zu finden. Fraktion VI enthielt die niedermolekularen Substanzen. Der Trypanblau-Test war in Fraktion I, IV und V positiv, in Fraktion II, III und VI negativ. Daraus ist zu schließen, daß sich die Entzündungsaktivität im Bereich der Albumine, $\alpha_{1}$ - und $\alpha_{2}$-Globuline befindet. Einen weiteren Beweis dafür erbrachten die Ergebnisse der Proteinfällung mit unterschiedlichen Ammoniumsulfatkonzentrationen. Die mit 34-proz. Ammoniumsulfat fällbaren Proteine enthielten nur $\beta$ - und $\gamma$-Globuline, der Trypanblau-Test war stets negativ. Das Elektropherogramm der Pleurapunktat-Proteine, die sich mit 40-proz. Ammoniumsulfat fällen ließen, enthielt zusätzlich $\alpha_{1^{-}}$und $\alpha_{2}$-Globuline; die Entzündungsaktivität war in $80 \%$ positiv. Mit 50 - und 60 -proz. Ammoniumsulfat konnten alle Proteinfraktionen gefällt werden; der Trypanblau-Test waț in allen Fällen positiv.

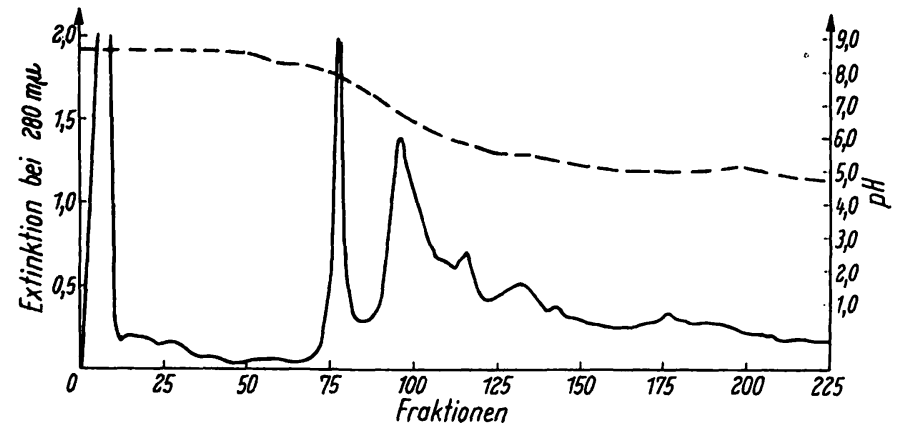

Abb. 2

Trennung von Pleurapunktat-Proteinen an Sephadex A-25.

Säule: $1,4 \times 15 \mathrm{~cm}$; aufgetragene Menge: $20 \mathrm{~m} /$ Punktat ; Elutionsmittel: $0,04 m-0,35 m$ Tris-Phosphatpuffer

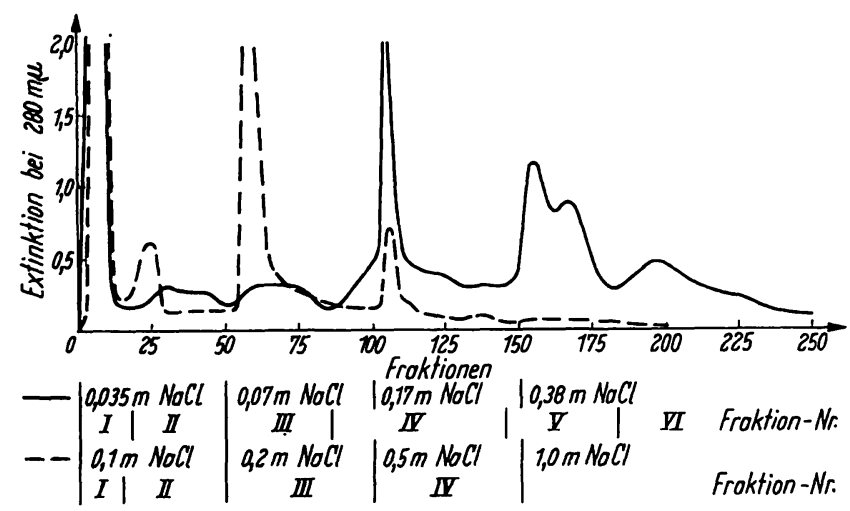

Abb. 3

Trennung von Pleurapunktat-Proteinen an Sephadex A-25.

Säule: $1,4 \times 15 \mathrm{~cm}$; aufgetragene Menge: $20 \mathrm{ml}$ Punktat; Elutionsmittel: $0,035 m-0,38 m \mathrm{NaCl}$

- - Elutionsmittel: $0,1 m-1,0 \mathrm{~m} \mathrm{NaCl}$

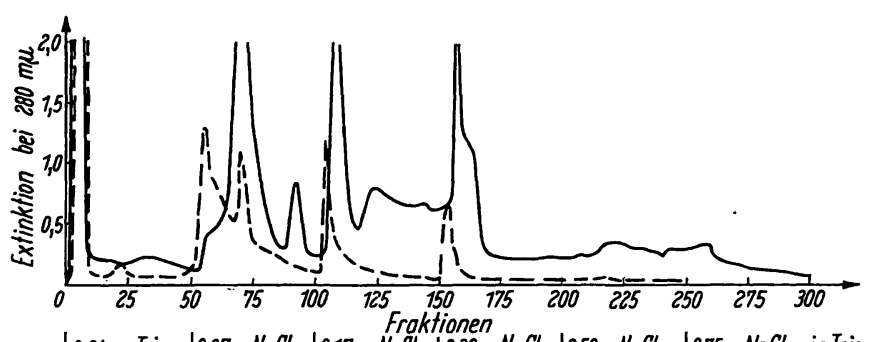

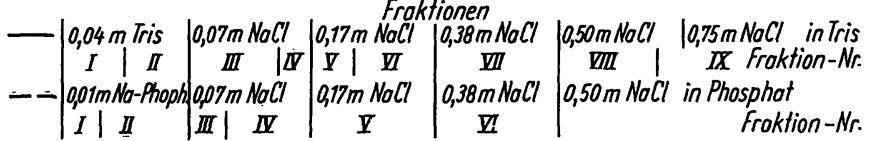

Abb. 4

Trennung von Pleurapunktat-Proteinen an Sephadex A-25. Säule $1,4 \times 15 \mathrm{~cm}$; aufgetragene Menge: $20 \mathrm{ml}$ Punktat; ...... Elutionsmittel: $0,035 \mathrm{~m}-0,75 \mathrm{~m} \mathrm{NaCl}$ in $0,04 \mathrm{~m}$ Tris-Puffer, $\mathrm{pH} \mathrm{8,6}$,

- - - Elutionsmittel: $0,035 \mathrm{~m}-0,38 \mathrm{~m} \mathrm{NaCl}$ in $0,01 \mathrm{~m}$ Natriumphosphatpuffer, pH 8,6

\section{Literatur}

1. LEwrs, T., The blood vessels in the human skin and their responses. Shaw and sons, London (1927). - 2. MENKIN, V., Int. Arch. Allergy 4, 131 (1953). - 3. Schaugr, A., Münch. med. Wschr. 106, 799 (1964). - 4. Frodin, P., Dextran Gels and their Applications in Gel Filtration, Diss. Univ. Uppsala (1962). - 5. Porath, J. und P.Flodis, Nature, (London) 183, 1657 (1959). - 6. Corn, E. J., T. L. McMeekins, J. L. Onclex, J. M. Neveli und W. L.
Hughes, J. Amer. chem. Soc. 62, 3386 (1940). - 7. Grassmann, W. und K. Hannig, Hoppe-Seyler's Z. physiol. Chem. 290, 1 (1952). - 8. Emmrich, R. und S. KöNig, Med. Klin. 56, 661 (1961). - 9. Eммrich, R., Zschr. inn. Med., Leipzig 19, 8 (1964). - 10. Peterson, E. A., M. M. Wyckhoff und H. A. Sober, Arch. Biochem. Biophysics 93, 428 (1961). 